\title{
Kernel Flux: a whole-head 432- magnetometer optically-pumped magnetoencephalography (OP-MEG) system for brain activity imaging during natural human experiences
}

Ethan Pratt, Micah Ledbetter, Ricardo Jiménez-Martínez, Benjamin Shapiro, Amelia Solon, et al.

Ethan J. Pratt, Micah Ledbetter, Ricardo Jiménez-Martínez, Benjamin Shapiro, Amelia Solon, Geoffrey Z. Iwata, Steve Garber, Jeff Gormley, Dakota Decker, David Delgadillo, Argyrios T. Dellis, Jake Phillips, Guhan Sundar, Jerry Leung, Jim Coyne, Mike McKinley, Gilbert Lopez, Scott Homan, Lucas Marsh, Mary Zhang, Vincent Maurice, Benjamin Siepser, Teresa Giovannoli, Brandon Leverett, Gabriel Lerner, Scott Seidman, Vicente DeLuna, Kayla Wright-Freeman, Julian Kates-Harbeck, Teague Lasser, Hooman Mohseni, T. J. Sharp, Anthony Zorzos, Antonio H. Lara, Ali Kouhzadi, Alejandro Ojeda, Pronoy Chopra, Zachary Bednarke, Michael Henninger, Jamu K. Alford, "Kernel Flux: a whole-head 432-magnetometer optically-pumped magnetoencephalography (OP-MEG) system for brain activity imaging during natural human experiences," Proc. SPIE 11700, Optical and Quantum Sensing and Precision Metrology, 1170032 (5 March 2021); doi: 10.1117/12.2581794 


\title{
Kernel Flux: a whole-head 432-magnetometer optically-pumped magnetoencephalography (OP-MEG) system for brain activity imaging during natural human experiences
}

Ethan J. Pratt*, Micah Ledbetter, Ricardo Jiménez-Martínez, Benjamin Shapiro, Amelia Solon, Geoffrey Z. Iwata, Steve Garber, Jeff Gormley, Dakota Decker, David Delgadillo, Argyrios T. Dellis, Jake Phillips, Guhan Sundar, Jerry Leung, Jim Coyne, Mike McKinley, Gilbert Lopez, Scott Homan, Lucas Marsh, Mary Zhang, Vincent Maurice, Benjamin Siepser, Teresa Giovanolli, Brandon Leverett, Gabriel Lerner, Scott Seidman, Vicente DeLuna, Kayla Wright-Freeman, Julian Kates-Harbeck, Teague Lasser, Hooman Mohseni, T.J. Sharp, Anthony Zorzos, Antonio H. Lara, Ali Kouhzadi, Alejandro Ojeda, Pronoy Chopra, Zachary Bednarke, Michael Henninger, Jamu K. Alford

Kernel, Culver City, California.

\begin{abstract}
MEG based on optically-pumped magnetometry (OP-MEG) operates with miniaturized, wearable insulation, in contrast to massive cryogenic dewars for SQUID-MEG, and allows placement of the sensors close to the scalp. This allows more natural head motion during data recording and localized signal quality comparable to, or surpassing, SQUID-MEG. However no OP-MEG system to date has offered full-head coverage with dense sensor packing, and existing systems - as with SQUID-MEG require the subject to be sealed in a multilayer, passively-shielded vault in order to suppress ambient magnetic fields. Here we present Kernel Flux, which overcomes these limitations. Kernel Flux uses a collection of alkali vapor sensors in a unique array architecture to directly detect the magnetic fields generated by collective neural activity in the brain, while allowing for comfortable head motion. Each Kernel Flux OP-MEG system was designed from the ground up to work as an integrated system optimized around the user's experience, with relevance to natural home and office contexts.
\end{abstract}

Keywords: Kernel, OP-MEG, OPM, alkali vapor cell, Kernel Flux, MEG, magnetoencephalography, functional brain imaging

*ethan.pratt@kernel.com

Optical and Quantum Sensing and Precision Metrology, edited by Selim M. Shahriar, Jacob Scheuer,

Proc. of SPIE Vol. 11700, 1170032 - CThe Authors. Published under a Creative Commons

Attribution CC-BY 3.0 License · doi: 10.1117/12.2581794 


\section{INTRODUCTION}

Magnetoencephalography (MEG) is a non-invasive, functional neuro-imaging technique, which uses magnetometers near the surface of the scalp to measure magnetic fields arising directly from the activation of neurons in the brain. Neuromagnetic fields exit the scalp while retaining the pristine signatures of their causally direct brain activity, because magnetic fields pass through bone and tissue with negligible distortion [1,2]. Outside the head, these brain signals can be recorded by arrays of magnetometers to provide functional neuroimages with high spatial resolution, along with sufficient bandwidth to capture fluctuations, on the order of milliseconds, of a full spectral range of healthy human neural oscillations up to hundreds of $\mathrm{Hz}$. These features make MEG a critical tool for studying brain activity $[\underline{3}, \underline{4}]$.

Traditional MEG sensors are based on superconducting quantum interference devices (SQUIDs). These systems suffer from very high capital cost and facility infrastructure barriers due to the size and inflexibility of their cryogenic dewars, along with the massive multilayered magnetically-shielded rooms typically required for their operation. Recent developments in optically pumped magnetometer (OPM) technology promise to significantly lower these cost and infrastructure barriers $[5, \underline{6}, \underline{7}, \mathbf{8}]$. OP-MEG sensors are based on compact atomic vapor cells in lightweight packaging that can be worn on - and move with - the participant's head $[\underline{9}, \underline{10}, 11,12]$. With small $(<1 \mathrm{~cm})$ scalp-to-sensor distances and up to three dimensional field vector measurements, OPMs offer rich MEG data that, even in prototype systems, can match and surpass the information density of SQUID-MEG systems [13,14,15]. Furthermore, compared to the extremely limited subject motion allowed in traditional SQUID-MEG systems, a wearable, co-moving sensor array offers a key advantage that dramatically expands the range of naturalistic human experiences to be studied for the first time, with the highest-fidelity brain function imaging at the full speed of human cognition.

\section{SYSTEM ARCHITECTURE}

Flux 1.0 is a whole-head magnetoencephalography system based on optically-pumped magnetometers. The OPMs are arranged in a modular format, which allows for adjustability of the sensors to suit various participant head sizes, while maintaining a typical scalp-standoff distance less than $8 \mathrm{~mm}$. The system includes a mechanical support structure that relieves weight and torque from the participant while allowing for a range of conversational head motion. A system overview is presented in Figure 1 (a). The magnetically shielded room provides a quiet background from which sub-picoTesla level neuromagnetic fields can be recorded. Segmenting the system, as shown, minimizes the number of reinforced cables that must be fed through the enclosure wall. 


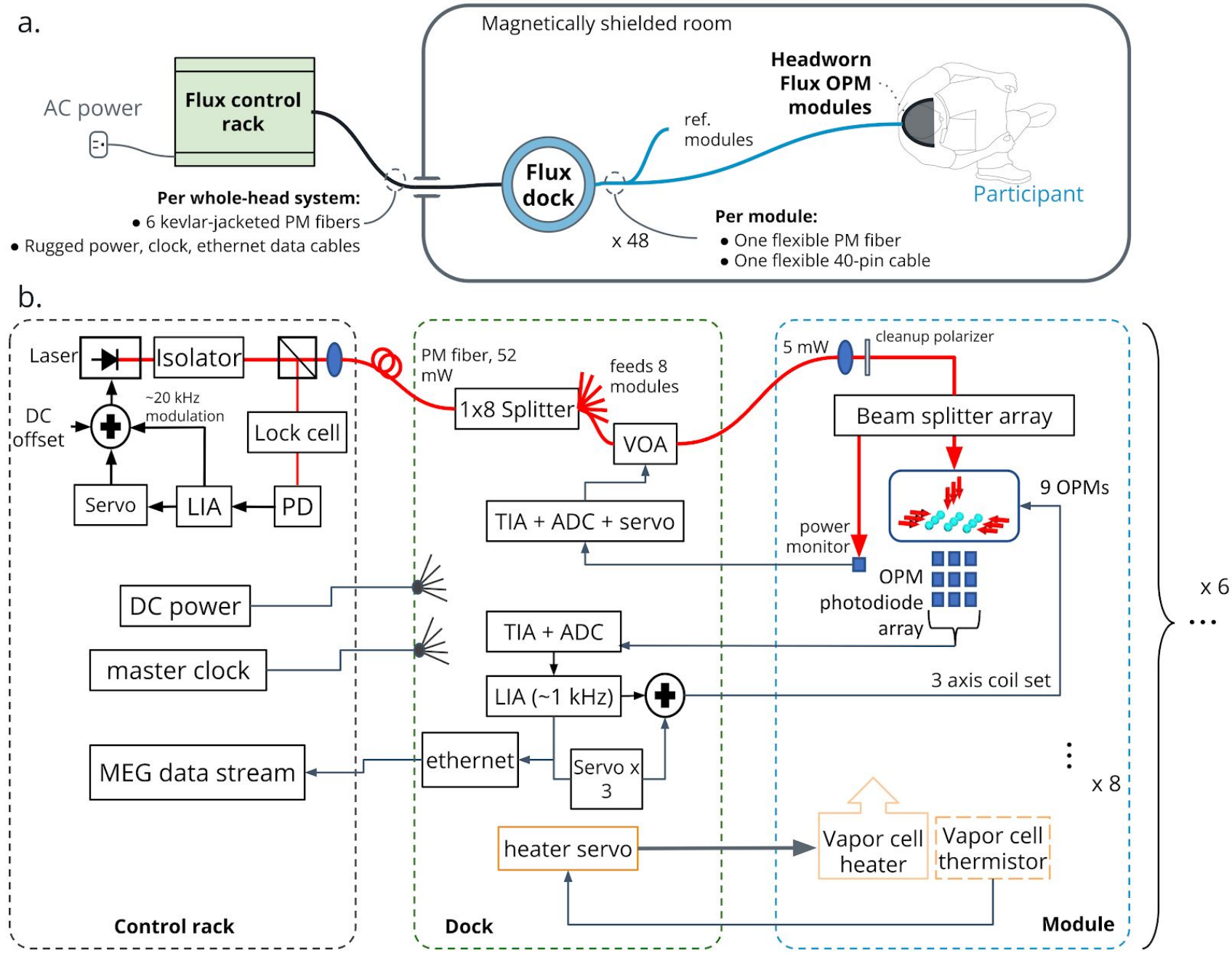

Figure 1. Flux system diagram. (a) A participant wearing a helmet populated with Flux modules is able to interact with an audio-visual environment inside of a magnetically shielded room. Typically up to eight "ref. modules," fixed with respect to the room, can be used as reference sensors. The Flux Dock, in addition to housing various electronics to operate the sensors, provides mechanical support for the helmet.. (b) Laser system, control loops, and signal processing chain that comprise the Flux optically-pumped MEG system. PM: Polarization maintaining. LIA: Lock-in amplifier. PD: Photodiode. TIA: Trans-impedance amplifier. ADC: Analog-digital converter.

The laser system, control loops, and signal processing chain that comprise the optical system for Flux are shown in Figure 1(b). Light from each distributed feedback laser in the Control Rack passes through an optical isolator and beamsplitter before coupling into a fiber. In the control rack, light picked off by the beam splitter passes through a warm reference vapor cell $\left(\sim 80^{\circ} \mathrm{C}\right)$ and can be used to frequency lock the laser to the peak of the $\mathrm{Rb}$ absorption by monitoring power transmitted through the vapor cell at the first harmonic of a small $20 \mathrm{kHz}$ modulation applied to the laser drive current. Within the Dock, the laser is distributed to 8 independent optical fibers, which contain in-line variable optical attenuators 
(VOAs) used to stabilize laser intensity measured with an in-module monitor photodiode illuminated by light that does not pass through the vapor cells. Optical pumping beams are circularly polarized while interacting with $\mathrm{Rb}$ vapor in a double-pass configuration, followed by detection at an array of nine photodiodes. Their photocurrents are amplified, digitized, and analyzed via lock-in detection in the Dock. Two phase-resolved photocurrent quadratures detected at the first harmonic of the magnetic modulation frequency serve as measures of the two magnetic field components orthogonal to each OPM optical beam. Our sensor can be operated in open or in closed loop by feeding the demodulated signals into a three axis servo that drives DC current through the onboard three-axis coil set.

\section{Whole-head sensor configuration}

The placement of 360 Flux OPMs, comprising 40 modules, is illustrated by the montage plot in Figure 2. As is described in detail below, the unique sensor architecture enables the close packing of modules around the head without compromising sensitivity due to cross-talk.

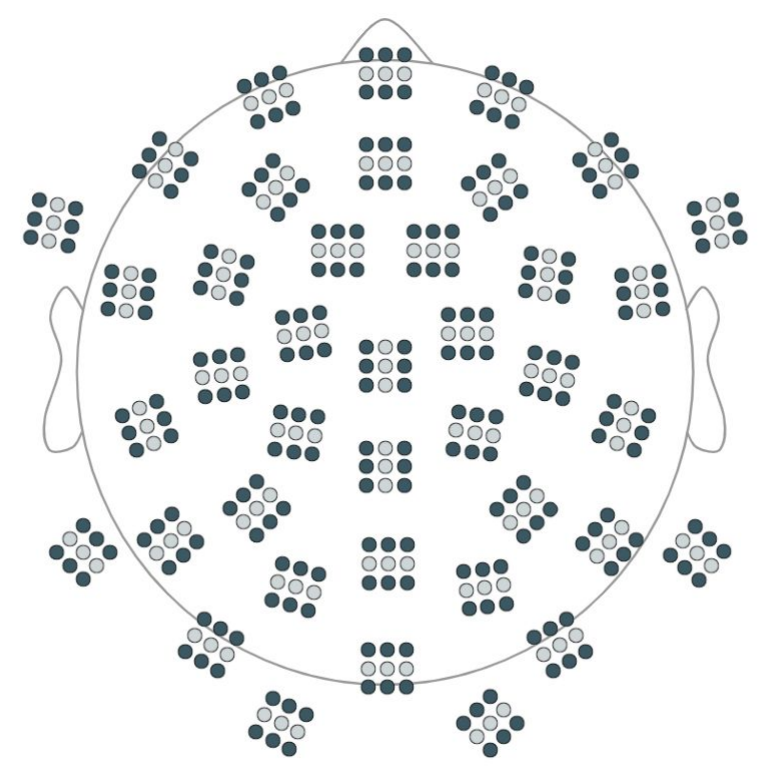

Figure 2. Flattened montage illustrating whole-head sensor placement. 240 dark-colored OPMs are each sensitive to the locally scalp-radial field plus one scalp-tangent component (labeled $B z$ and $B y$ by convention). 120 light-colored OPMs are sensitive to the two locally scalp-tangent components By and $B x$.

\section{PHYSICS OF OPERATION}

Each Flux OPM is a single-beam, spin-exchange relaxation free (SERF) mode, near-zero-field vector magnetometer composed of an optically-pumped volume of Rubidium-87 atoms in a microfabricated vapor cell $[16,17]$. An OPM's fundamental responsivity to magnetic field derives from a well-known magnetic resonance exhibited by optically-pumped alkali atoms [18]. The nine OPMs in a Flux module share a common, stabilized $795 \mathrm{~nm}$ diode laser source in the control rack, whose optical pumping light 
is delivered via fiber to the module. There, the light is collimated and distributed to the 9 vapor cells in each module, which are arranged in a $3 \times 3$ square array with $5 \mathrm{~mm}$ spacing. Light entering each vapor cell is circularly polarized with a quarter-wave plate, and reflected through the vapor cell, resulting in a double-pass configuration, where the counter-propagating light beams have opposite helicity. In such a configuration, atoms in the double-passed beam are continuously pumped into the maximally stretched angular momentum state of the ${ }^{2} \mathrm{~S}_{1 / 2}$ ground state.

As magnetic fields in the plane orthogonal to the pumping axis are scanned through zero amplitude, the transparency of atomic vapor exhibits a Lorentzian response [19]. The amplitude and full-width, half-maximum (FWHM) of this response determines the responsivity of the OPMs, the latter of which is related to the relaxation rate of atoms from their pumped state via a number of processes, including: diffusion of atoms, spin-exchange collisions, collisions with the wall of the vapour cell and magnetic field inhomogeneities [20], with spin-exchange collisions being the dominant effect [21].

The vapor cells are maintained at approximately $120^{\circ} \mathrm{C}$ by onboard heaters in the modules, which ensures sufficient $\mathrm{Rb}$ vapor density to suppress relaxation due to infrequent spin-exchange collisions, thereby enabling the femtoTesla (fT) sensitivity required for MEG [22,23]. The module components are insulated to maintain comfortable skin-contact temperature.

Flux modules include an integrated local three-axis, planar coil set built on printed circuit boards for high-uniformity and repeatability. These coils provide local background field compensation, as well as magnetic field amplitude modulation at all 9 OPMs. Each coil set is calibrated using scalar field resonances recorded at each OPM [24,25]. In this operation mode, optical absorption features occur at a magnetic field value that can be calculated independently of the coil parameters.

The coils are used to modulate magnetic fields along all three module-frame axes at each OPM at 2200 $\mathrm{Hz}$, providing sensitivity along axes orthogonal to the optical pumping beam. Changes to the magnetic field within the optically pumped vapor - including changes caused by time-varying neuromagnetic fields - result in detectable changes to the partial transparency of the rubidium vapor. This causes corresponding changes in the amplitude of the residual pump light, which is recorded by one photodiode per OPM in the module. Its photocurrent is amplified, digitized, and demodulated with precise timestamping in the dock.

A single OPM modulated in this way is sensitive to magnetic field vectors along two responsive axes, each orthogonal to the optical pumping beam. Because the module arranges 6 OPMs with beams tangential to the scalp, plus 3 OPMs with beams normal to the scalp, the module provides cumulative 3-axis response ( 6 channels of $\boldsymbol{B}_{z}: 3$ channels of $\boldsymbol{B}_{\boldsymbol{x}}: 9$ channels of $\boldsymbol{B}_{\boldsymbol{y}}$ ). The beams, local scalp orientation, and responsive vectors within a module are illustrated schematically in Figure 3. 


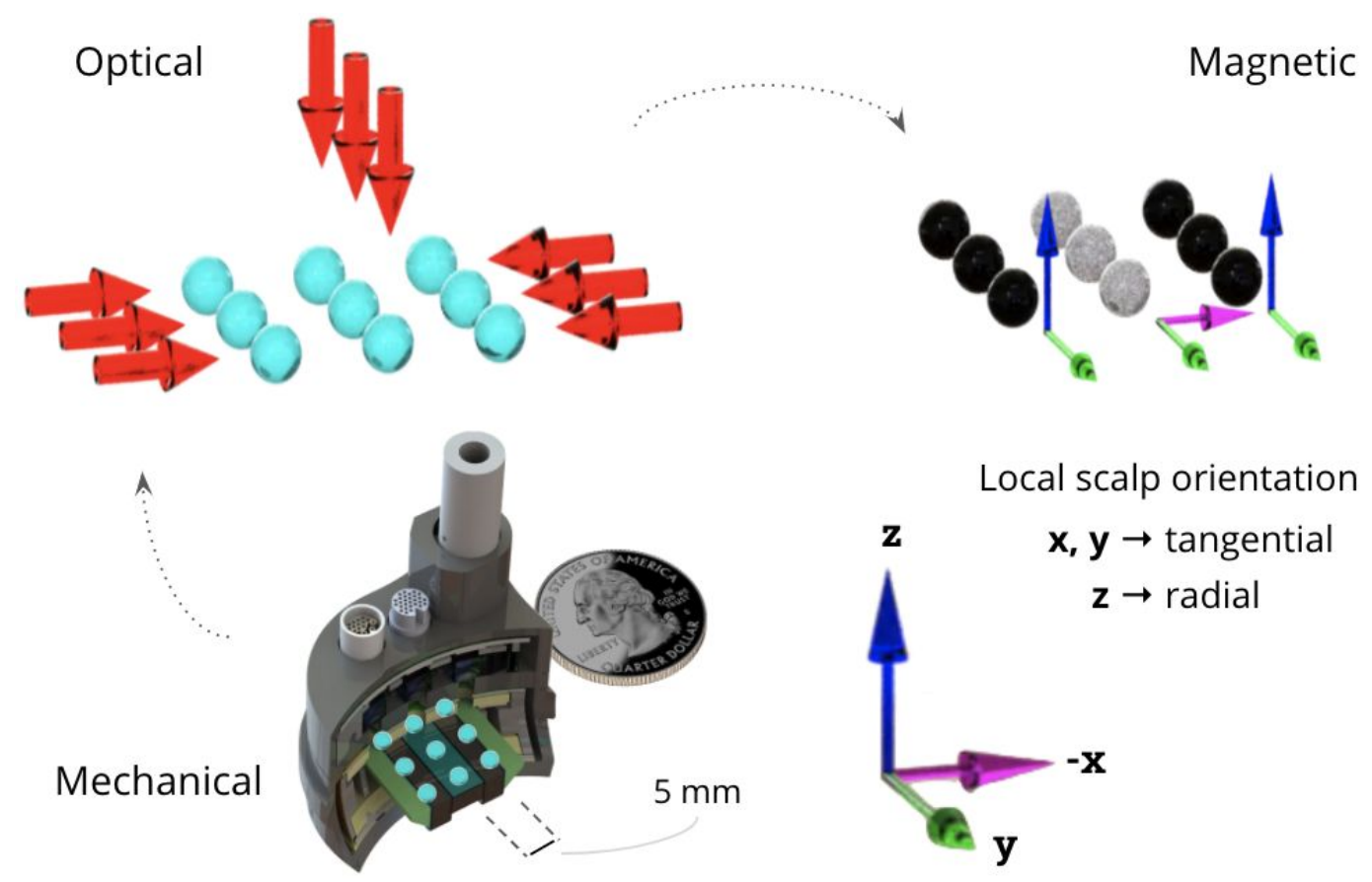

Figure 3 : Schematic of the OPM arrangement within a Flux module, showing the mechanical position of the OPMs within a module, the optical arrangement of light entering each OPM, and the magnetically sensitive axes of each OPM. Light blue spheres represent the sensitive volumes of the atomic vapor. Large red arrows represent the incoming light to each OPM. The optical beam axis in each OPM determines the transverse magnetically sensitive axes. In the reference frame of the scalp, the indicated $\mathbf{x}$ and $\mathbf{y}$ axes are tangential to the scalp and the $\mathbf{z}$ axis is radial.

\section{PERFORMANCE}

\section{Sensitivity}

To evaluate the magnetic sensitivity of the Flux sensors, modules were placed in a magnetically shielded environment and operated in single-axis sensitivity mode (field modulation along a single axis). The first harmonic of the demodulated output from the photodiode signals was recorded for 10 seconds. A calibration tone from the on-board coils provides a reference for scaling the magnetic field data. The power spectral density of the data, normalized to the frequency response of the magnetometer, records the spectral noise characteristics.

Sensitivities at neurally-relevant frequency bands are shown in Table 1, for three signal processing algorithms. These algorithms illustrate the use of tailored averaging to optimally resolve a target neuromagnetic field, using knowledge of the configuration of Flux OPMs in modules. This balances the natural tradeoff between the spatial curvature of fields that can be sampled by a given pitch of effective sensors in an array, and the number of effective sensor channels produced by signal processing algorithms that use averaging across OPMs to reduce noise. 


\begin{tabular}{|c|c|c|c|c|c|c|c|}
\hline & & $\begin{array}{c}3 \mathrm{~Hz} \\
\delta\end{array}$ & $\begin{array}{c}11 \mathrm{~Hz} \\
\mathrm{a}\end{array}$ & $\begin{array}{c}23 \mathrm{~Hz} \\
\beta\end{array}$ & $\begin{array}{c}37 \mathrm{~Hz} \\
\gamma\end{array}$ & $\begin{array}{l}71 \mathrm{~Hz} \\
\text { High-y }\end{array}$ & $\begin{array}{l}200 \mathrm{~Hz} \\
\text { High-y }\end{array}$ \\
\hline Signal processing mode & Channel count & \multicolumn{6}{|c|}{ Sensitivity at each noted frequency $(\mathrm{fT} / \sqrt{ } \mathrm{Hz})$} \\
\hline Independent OPMs & 720 & 80 & 33 & 30 & 25 & 25 & 30 \\
\hline Mean-subtracted & 720 & 50 & 25 & 22 & 20 & 20 & 22 \\
\hline Module-averaged & 120 & 25 & 20 & 18 & 16 & 16 & 20 \\
\hline
\end{tabular}

Table 1: Magnetometer sensitivities for three different choices of signal processing algorithm. Whole-head channel count, for both Independent OPM processing and mean-subtraction: 40 modules $\times 9$ OPMs $\times 2$ axes $=720$. Whole head channel count for the module-averaged processing: 40 modules $\times 3$ axes $=120$. Note that for the purposes of sensor-space characterization, we define a channel as a time-series representation of one vector component of the magnetic field at a location outside the head.

Note that there is no change to the system operation or raw data due to these or other downstream processing options. Independent OPMs: OPM sensor channels are reported without spatial averaging. This provides highest channel count, and smallest effective sensor pitch. Mean-subtracted: OPM sensor channels are treated independently after subtraction of the vector-resolved module mean. This reduces the effects of spatially uniform ("common-mode") fields, which helps resolve spatially intricate neuromagnetic signals that vary significantly between adjacent OPMs. Relatively uniform magnetic fields - which often arise from noise sources, but can also include fields due to neural activity - will be attenuated. Module-averaged: Vector-resolved sensor channels are averaged for all OPMs within each module, so that each module provides one effective three-axis measurement of the average field. This optimizes for sensitivity by increasing the cumulative number of measurements, with a longer effective baseline and fewer channels.

The fiber-coupled, high powered edge-emitting laser sources are a key architectural choice of this system. To understand why high optical power is advantageous for a whole-head OPM array with expanded head motion, consider the performance tradeoff illustrated in Figure 4. Here we show the equivalent magnetic noise of a Flux OPM - along with the power-broadened linewidth of the magnetic resonance - as a function of optical power. In this regime, increased optical pumping leads to an increase in both the linewidth and amplitude of the magnetic resonance. As a result, the responsivity of the magnetometer is constant, which allows for an increase in bandwidth (or, equivalently, open-loop dynamic range) while holding magnetic sensitivity substantially constant. 


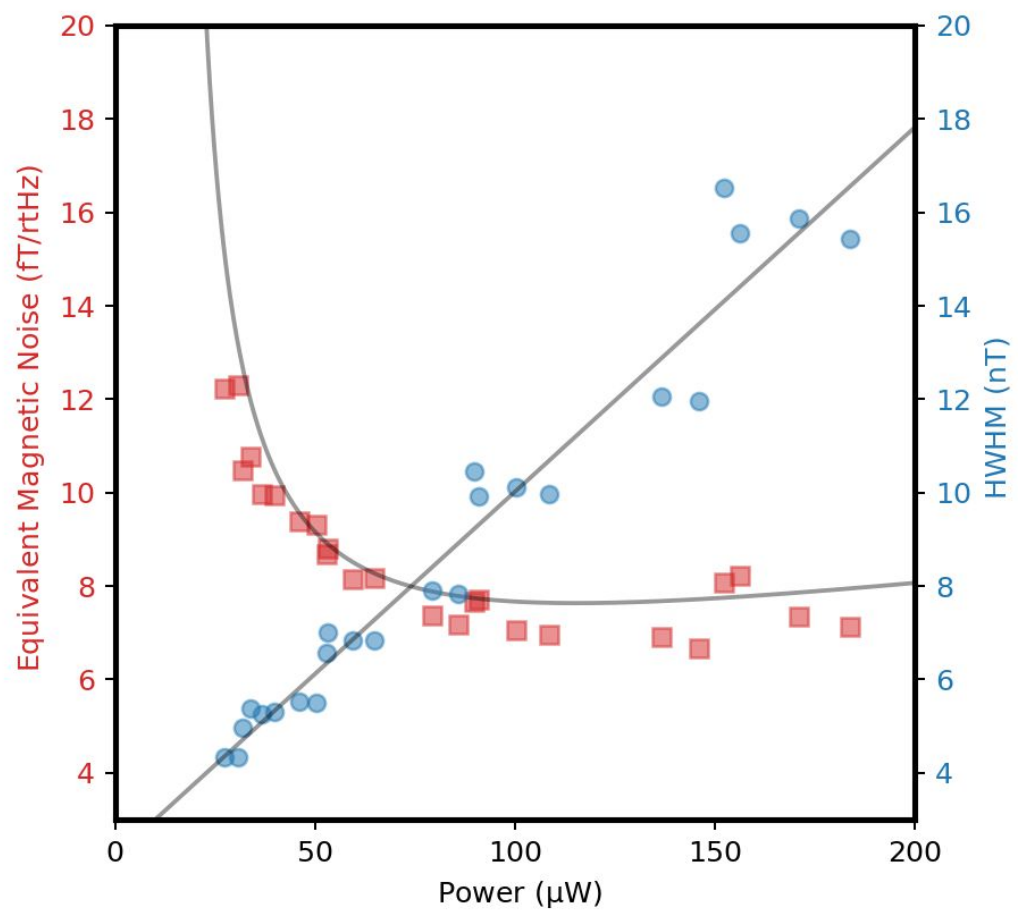

Figure 4: Comparison of magnetic noise floor driven by photon shot noise, and the half-width-half-max (HWHM) of the magnetic resonance, as a function of optical power entering a Flux OPM in a passive desktop shield. As optical power increases above $70 \mu \mathrm{W}$, the linewidth of the magnetic resonance increases linearly - driving up the open-loop dynamic range - without significantly degrading the net equivalent magnetic noise.

In addition, as long as each OPM is sufficiently illuminated, the 9 OPMs in each Flux module array provide for cancellation and/or decorrelation of excess common-mode optical intensity noise. Flux modules include a cleanup polarizer and a monitor photodiode, which picks off a fraction of the input light at each module and enables a closed-loop intensity servo. Taken altogether, these factors suggest that several $\mathrm{mW}$ light is optimal for the input to each module, which is best achieved by edge-emitting sources coupled via fiber. A whole-head array of many hundreds of Flux OPMs can be efficiently driven by just a few high-powered fiber-coupled sources.

\section{Dynamic range: MEG in motion}

A key advantage of wearable MEG instrumentation over fixed SQUID systems is that subject motion does not alter the head to sensor-array co-registration, because the head and sensor-array are co-moving. However, subject motion during wearable MEG measurements can result in magnetometer responses arising from the motion of the sensor in a background magnetic field, even if that field is static. In the moving-head frame, these non-neural fields appear as if from a distant fluctuating source.

The capacity of a magnetometer array to distinguish nearby signal sources from distant noises is illustrated by the system's common mode rejection ratio (CMRR). For Flux, a key measure is therefore 
the CMRR within each intrinsic array module, as illustrated by the $\boldsymbol{B}_{\boldsymbol{y}}$ measurements in Figure 5 (a). These data were recorded under conditions of an artificially intense (for spectral clarity), spatially-uniform fluctuating field applied to a module, which emulates sensor-array motion with no human participant.
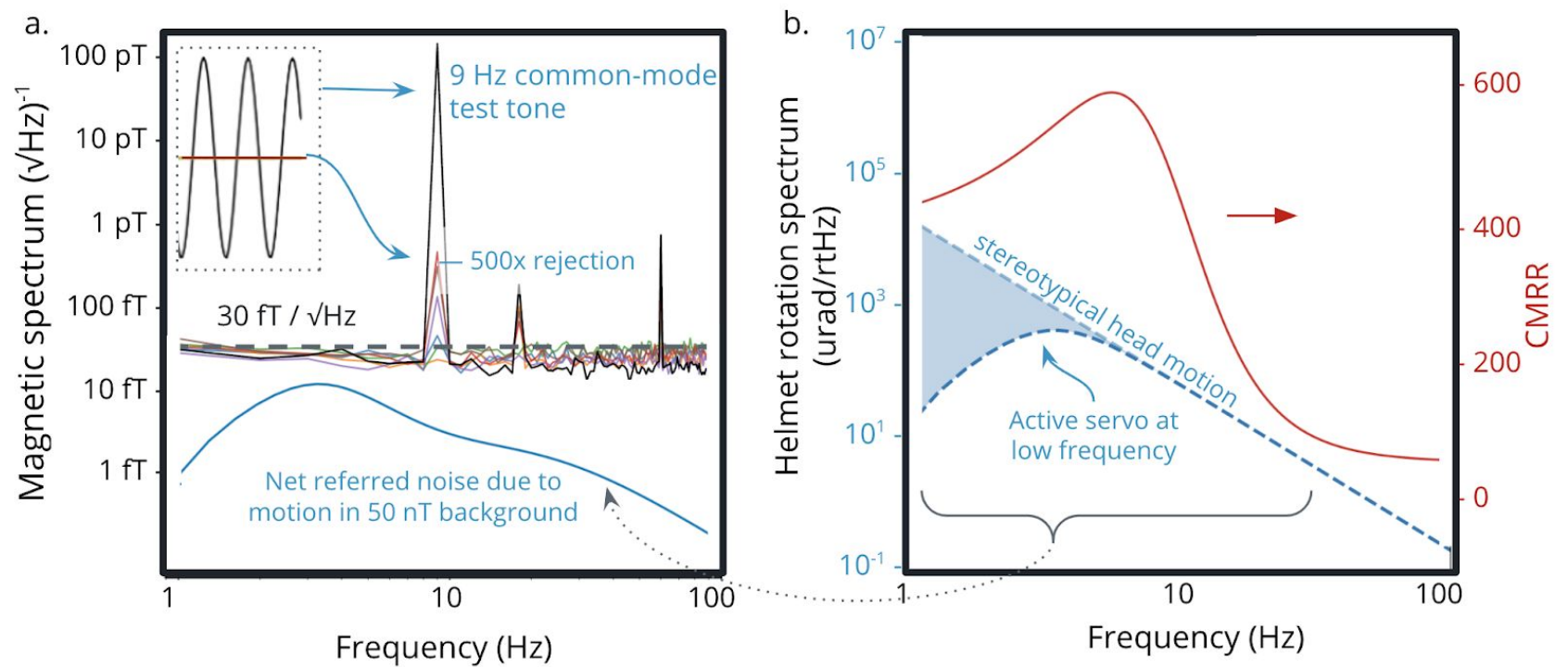

Figure 5: Common mode rejection of spatially homogeneous signals on the Flux module, and active servoing at low frequencies enables head motion during OP-MEG. (a) Magnetic noise spectral density along a single OPM axis while a $9 \mathrm{~Hz}$ sinusoidal tone is applied to a module. Inset shows a representative time series segment of the data. The black trace shows the mean of the 9 OPM outputs. The colored traces are the mean-subtracted signals from each OPM, showing at least 500x rejection of the common mode $9 \mathrm{~Hz}$ tone. The lower blue trace represents the expected noise floor contribution from stereotypical head motion in a $50 \mathrm{nT}$ background field, after low frequency servoing and common mode rejection. (b) Spectrum of stereotypical head motion (dashed light blue power law) is compensated at low frequency with closed loop operation (dashed dark blue) of the Flux module coils. The measured common mode rejection ratio (CMRR) of a flux module is plotted in red. Together, and assuming a $50 \mathrm{nT}$ background field, these infer the net magnetic noise shown as the solid blue curve in (a).

In Figure 5 (b), the CMRR of within a module is plotted alongside a representative spectral dependence of head rotation. Conversational motion of a human head corresponds to head pose angle fluctuations of $1 \mathrm{mrad} / \sqrt{ } \mathrm{Hz}$ at $1 \mathrm{~Hz}$ timescales, to more rapid jitter motion, around $60 \mu \mathrm{rad} / \sqrt{ } \mathrm{Hz}$ at $9 \mathrm{~Hz}$. In the rapid motion case, fluctuations of $60 \mu \mathrm{rad} / \sqrt{ } \mathrm{Hz}$ in a $50 \mathrm{nT}$ background field would change the common-mode field projection on any module axis by less than $3 \mathrm{pT} / \mathrm{V} \mathrm{Hz}$. After common-mode rejection by a factor of 500, the residual artifact would be smaller than $6 \mathrm{fT} / \sqrt{ } \mathrm{Hz}$ - below the magnetic noise floor of the OPMs. The high common-mode rejection ratio in Flux modules is made possible by the design choice of closely packed OPMs within a module, sharing optical source, field modulation, and operating temperature.

For larger artifacts due to motion at lower frequencies, the operation range of a SERF magnetometer will be limited in large background magnetic field gradients, where the magnetometer may move into a field that saturates the magnetometer response, or move it into a non-linear regime. As a result, the 
operable dynamic range of most atomic magnetometer based MEG systems is limited below 5 nT $[7,12,15]$. Previous OP-MEG demonstrations have focused on using large, room-scale compensation coils to create spatially homogeneous fields in volumes $\sim 10 \mathrm{~cm}$ across to operate their system in, which allows for limited subject motion $[9,26,27]$. The local coil architecture of Flux enables local compensation of background fields in real-time, which provides a scalable solution for user-motion in elevated fields. In the Flux system, each module's three-axis magnetometer signals are fed back to the onboard coil current control. Since larger head-motion is mostly limited to timescales much longer than $200 \mathrm{~ms}$, motion artifacts in the magnetic signals are spectrally distinct from the dominant band of neural signals (>8 Hz). Therefore, the three-axis control loop for this feedback is optimized to operate at frequencies below $7 \mathrm{~Hz}$. In Figure 5(b), this active filtering is represented by the darker dashed curve .

This real-time, local compensation is made possible by intra-module 3-axis shield coils, whose fields are substantially homogeneous over the OPMs. The proximity of the coils to the vapor cells together with $<100 \mathrm{pA} / \sqrt{\mathrm{Hz}}$ noise levels in current drivers means that the magnetic noise contribution from the coils is $<10 \mathrm{fT} / \sqrt{\mathrm{Hz}}$ in any direction. In addition to head motion, the closed loop operation also addresses slow, transient signals that may arise from passing vehicles, moving objects, and other magnetic interference. Compensating the local background field at the magnetometers with the onboard coils means that Flux sensors have an effective dynamic range exceeding $200 \mathrm{nT}$ at low frequencies.

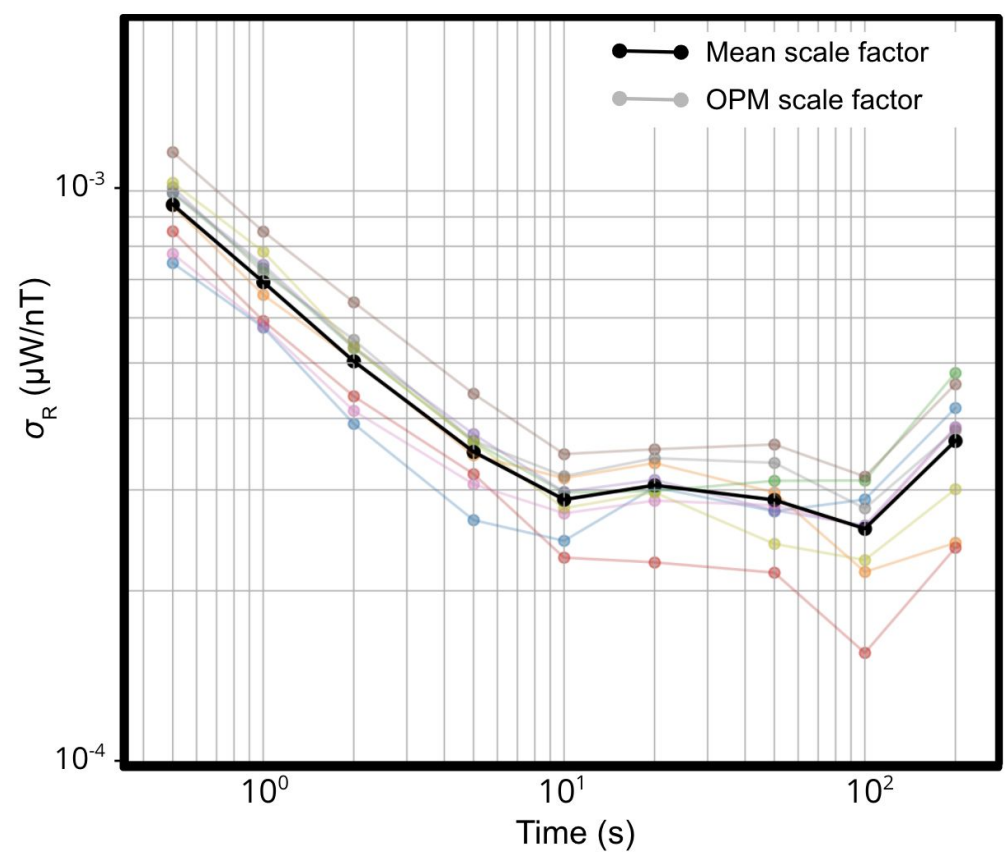

Figure 6 : Allan variance of magnetic field scale factors for magnetometer data from the 9 OPMs on a single module over 110 seconds. The mean variance, shown in black, is less than one part in 3000 between 10 and 100 seconds. 
Calibration factors that convert the demodulated response of the photodiode signals to magnetic field units can drift over time, affecting the CMRR performance and closed loop operation. Figure 6 presents the stability of magnetic field scale factors in Flux OPMs as shown by the Allan variance.

Figure 7 shows measurements during a phantom-tone experiment with large-scale apparent head motion in a $\sim 100 \mathrm{nT}$ background field. Again, the shared hardware elements of closely packed OPMs results in significant reduction in background noise, while highlighting gradient fields that arise from nearby sources. Through a combination of automatic cancellation of background fields, and gradiometry with high CMRR, the fT-range gradient at $22 \mathrm{~Hz}$ is recovered. The dynamic range of Flux is illustrated by the progression of scales from nT to pT to fT.

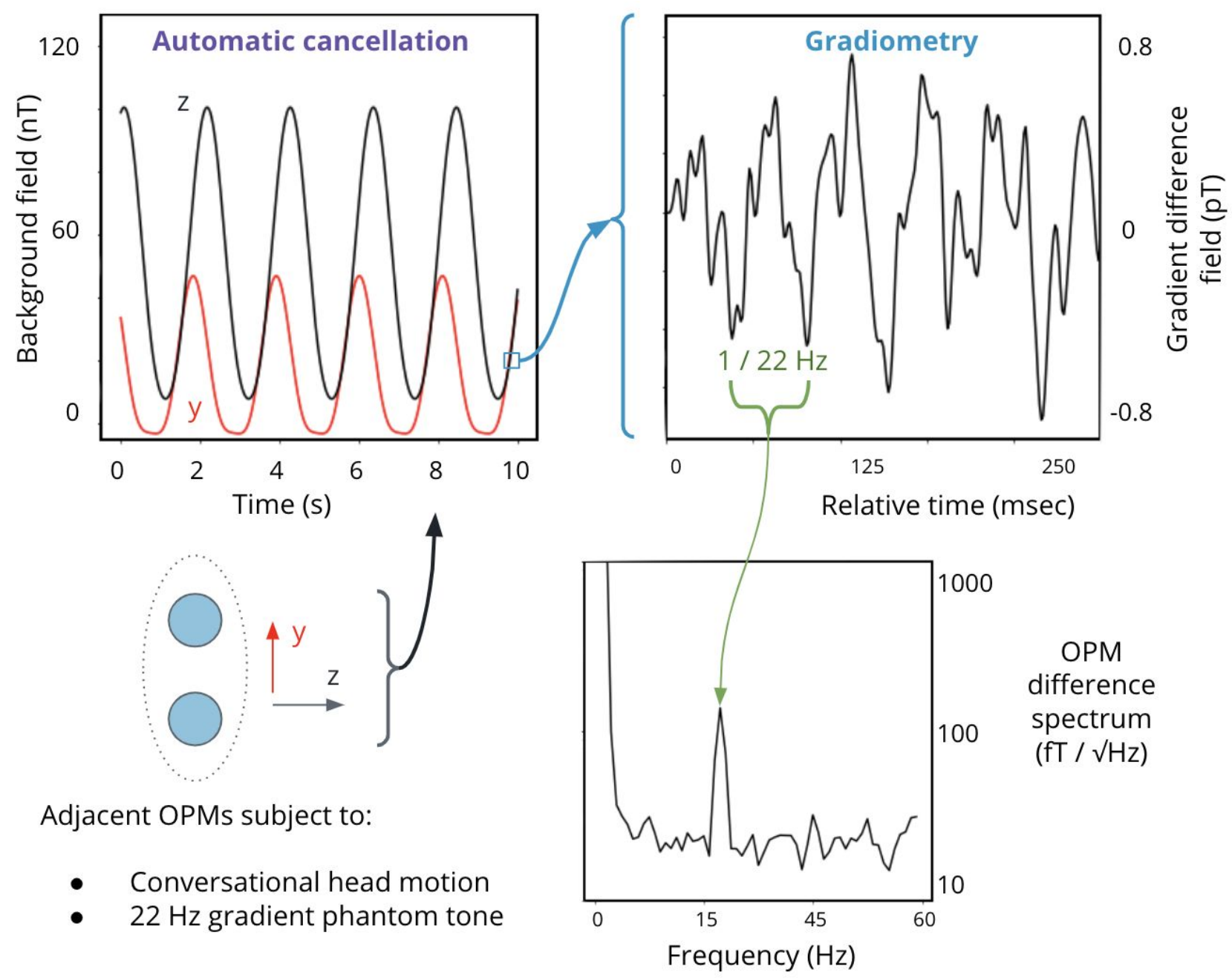

Figure 7: Data from a phantom experiment in which adjacent OPMs within a module are subjected to a $100 \mathrm{nT}$, sinusoidally varying background field at $2 \mathrm{~Hz}$, as well as a small, $22 \mathrm{~Hz}$ gradient phantom tone. The gradient field from the phantom tone mimics sources close to the sensor, while the spatially homogeneous background field mimics sources far from the head. Closed loop operation of the module and gradiometry between the two sensors, separated by $5 \mathrm{~mm}$, rejects the background fields, while the phantom tone at $22 \mathrm{~Hz}$ remains well resolved above the noise floor. 


\section{AUDITORY EVENT RELATED FIELDS}

MEG measures neural activity that occurs on millisecond timescales, enabling the study of neural activity as it occurs, such as decoding entrainment to long-form audio snippets [28], or for applications leveraging voluntary brain signals for real-time control of an interface [29,30]. One such signal with fast latency is the auditory event related field (ERF), whose peaks and troughs occur in the auditory cortex 0.1-0.2 s after the presentation of an abrupt auditory tone [31,32,33]. Due to its reliability and simplicity, it can be used as a test signal for MEG experiments [34]. Here we show magnetic fields recorded with Flux modules, that arose from neural responses from one participant who listened to roughly 200 short acoustic tones during a measurement session. Tones consisted of short acoustic pulses at $440 \mathrm{~Hz}$ at a comfortable volume, separated by variable intervals of 0.5-1 s, to allow for settling of the neural response before the next tone. The participant listened through both ears with non-magnetic earphones, and was seated in a magnetically shielded room (MSR), wearing non-magnetic clothing, and with no magnetic items on their person.

In standard ERF analysis, neural data are extracted around stimulus events, forming a series of trials, and averaged to increase the SNR of the response with respect to stochastic noise across trials. Prior to extracting and averaging trials, the Bz and By data from each of the outer 6 OPMs were downsampled to $100 \mathrm{~Hz}$, and bandpass filtered with an FIR filter between $0.5-20 \mathrm{~Hz}$. Additionally, we reduced environmental noise using regression, leveraging data from reference sensors which were offset from the head, and did not record any neural signal. Each trial consists of data between $0.2 \mathrm{~s}$ before, and 0.4 $s$ after stimulus onset.

In the lower right of Figure 8, we plot a trial-averaged ERF for a single OPM in the module. Plotting a birds-eye view of all trials recorded from this single OPM, in the upper right plot in Figure 8, we observe consistent single trial deflections around $0.1 \mathrm{~s}$ after the tone is played (tone is played at time $=0$ ).

In the left portion of Figure 8, we show module averaged ERFs for sensors, mapped onto the modules approximate location in the standard 10-20 system locations for EEG headsets. Each circle represents a sensor location, with " $\mathrm{F}$ " sensors being in the front of the head, and " $\mathrm{O}$ " sensors being on the back of the head. The sensors on the sides of the head, which are above the auditory cortex, show the strongest ERF responses.

The trial-averaged ERF response shows little qualitative variance across OPMs within a module - a result that is highlighted in the similarity between the single OPM example, and the module averages on the montage plot. The auditory ERF experiment evokes a spatially broad response across OPMs within modules located proximal to the auditory cortex. As a result, module-averaging can be a suitable choice for visualization and analysis. 


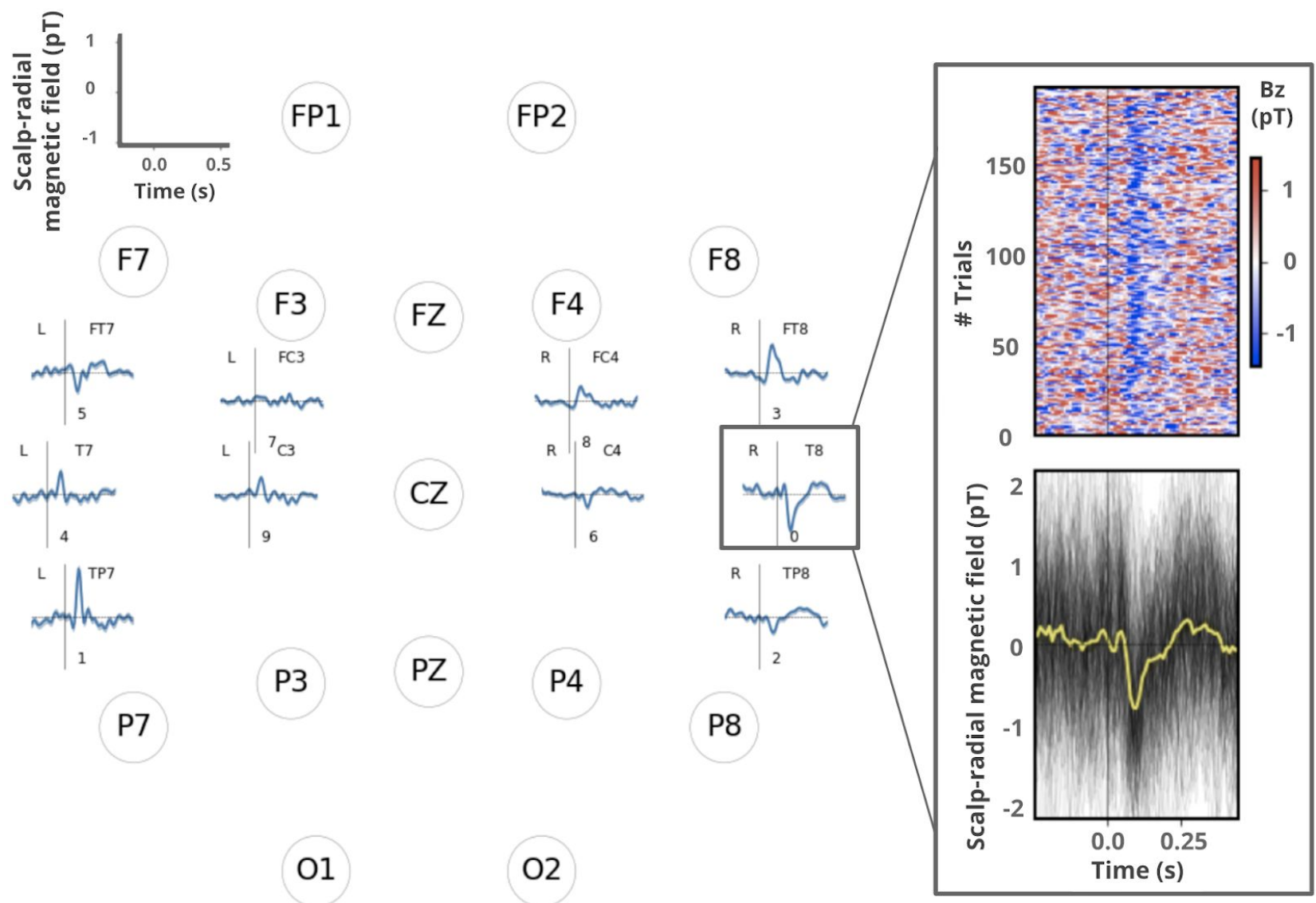

Figure 8: Left: Module averaged scalp-radial field (Bz in sensor space) recorded by Flux sensor modules, mapped onto the modules' approximated 10-20 EEG sensor locations. The modules located closest to the auditory cortex show the largest signals, from which detailed latency and spectral information can be extracted. Right: Example event related field (ERF) brain data from nearly 200 single trials observed following an auditory stimulus. The top and bottom plots display the same information in different graphical representations and share the same $x$-axis. In the top figure, the amplitude of the head-normal neuromagnetic field is color coded red/blue to represent positive and negative field direction, with units of picoTesla (pT) and a time axis reset to zero at every stimulus event. In the lower figure, each ERF is individually plotted (thin black solid lines) and the mean ERF is overlaid in yellow. For this auditory paradigm, a typical brain response generates an ERF peak onset following a delay of roughly $\sim 0.1 \mathrm{sec}$. The Flux system's capability to capture single-trial information is revealed by the visibility of vertical color bands in the top plot, and by the correlation between single trial responses and the ensemble average in the bottom plot. 


\section{PHANTOM SOURCES TO ILLUSTRATE SPATIAL RESOLUTION}

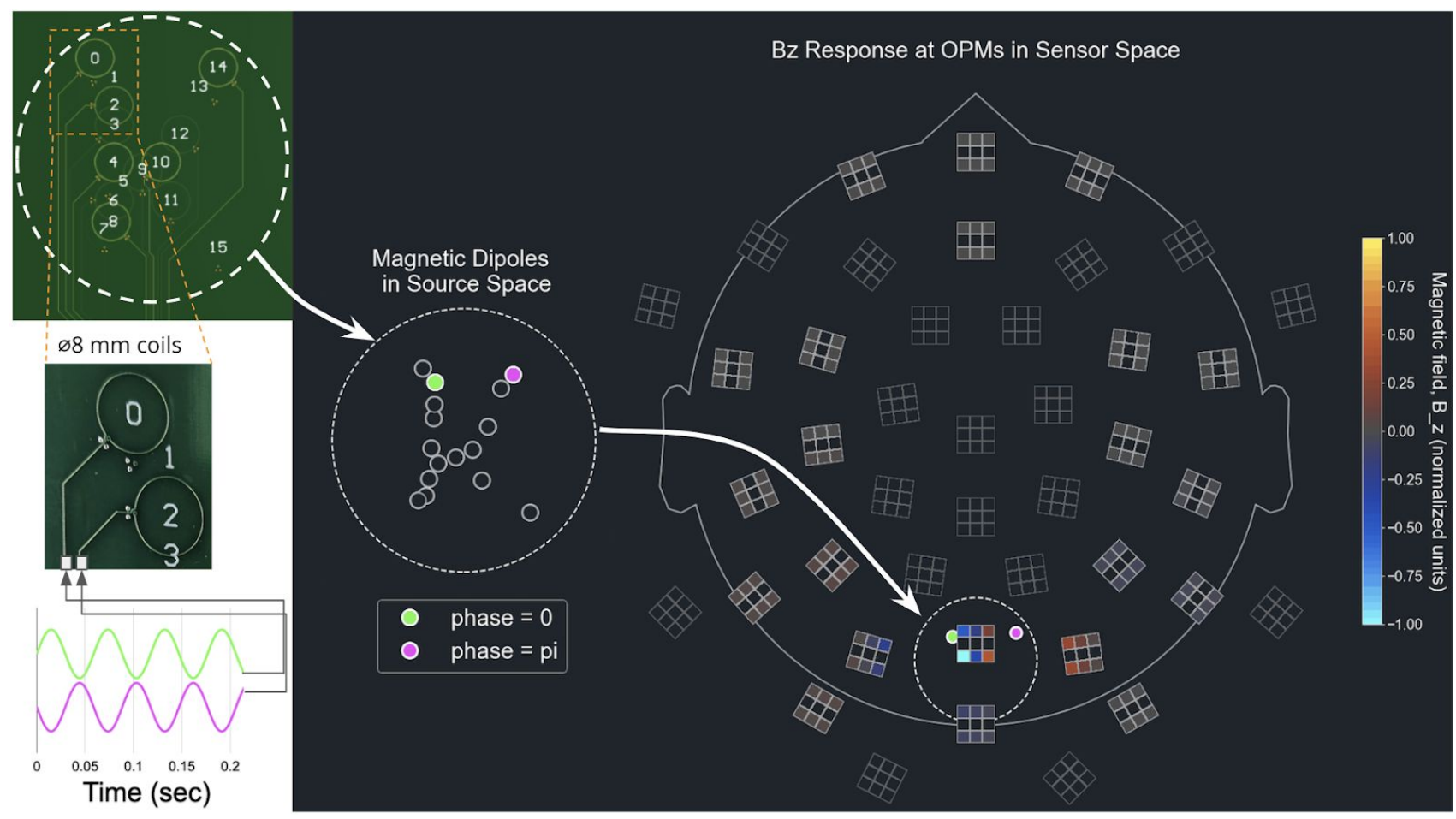

Video 1: Figure 9: Schematic overview of spatial resolution phantom experiment and results. Left bottom: Sinusoidal tones with opposite phase at $17 \mathrm{~Hz}$ drive $8 \mathrm{~mm}$ diameter wire loops on a printed circuit board, generating magnetic fields that mimic the field profile of a magnetic dipole. The phantom board is placed within the Flux helmet, approximately $2 \mathrm{~cm}$ from the nearest point in the helmet. Left top: The wire loops are arranged in a "K"-shaped pattern on the board. Center: The diagram shows magnetic dipoles in source space, schematically representing two loops that are activated. Right: 2D projection of the sensor space of the Flux helmet with position of the phantom board approximately indicated. The amplitude of the radial (Bz) magnetic response to the $17 \mathrm{~Hz}$ tones is shown for each OPM. Color bar scale is normalized to the maximum amplitude. Modules that are nearest to the active sources reveal magnetic field gradients at the OPM level, with phase offsets consistent with the respective sources.

\section{http://dx.doi.org/10.1117/12.2581794.1}

In addition to high temporal resolution, MEG with closely packed sensors can resolve fields from closely-separated sources. Here, we use synthetic signals to illustrate the spatial resolution of the Flux system. These synthetic validation signals provide a measure of ground truth, which cannot be guaranteed in the case of neural signals. Here, we present visualizations of our in-house developed resolution phantom system, which generates magnetic dipole field profiles by driving current through wire loops on a printed circuit board. Using this phantom, we are able to create simple or complex spatial patterns in the sensor space with any signal function. 
In Figure 9, a schematic of the resolution phantom board is shown in the top left. This schematic is diagrammed in the visualization in the center, where we can track the active phantom coils. The active coils shown are separated by $\sim 24 \mathrm{~mm}$, and the phantom board is placed inside of the Flux helmet, approximately $2 \mathrm{~cm}$ from the nearest point in the helmet. On the right is the projection of these sources onto the Flux sensor space, flattened for visualization onto a 2D head surface. Each square patch represents a module, and each sub square represents an OPM.

In this phantom experiment, we drive two separate coils with $17 \mathrm{~Hz}$ sinusoidal tones - one with a phase offset of 0 , and the other with a phase offset of $\pi$. The heatmap on the montage plot visualizes the measured Bz magnetic field amplitude of each OPM's response at $17 \mathrm{~Hz}$, normalized to the helmet-wide maximum amplitude. Modules that are not present in the headset, and OPMs that are not sensitive to Bz signals are greyed out. This procedure captures the phase relationship and amplitude of the sensor space signals with respect to a $17 \mathrm{~Hz}$ sinusoid reference signal with a phase offset of 0 . The nearest modules to the respective sources exhibit overall responses that are consistent with the phase offsets, and importantly, reveal clear gradients within the magnetic field profile.

\section{HUMAN SUBJECT DATA USED IN THIS MANUSCRIPT}

De-identified data used in this analysis were collected as part of internal research and product development activities using employee volunteers. This analysis was determined to be exempt from IRB review by WCG IRB. Employees who voluntarily participated provided their written informed consent prior to their participation.

\section{FUNDING AND COMPETING INTERESTS}

This work was funded by Kernel.

\section{ACKNOWLEDGMENTS}

The authors would like to thank Katriece Ray for shaping our participant experience, Nick Cammorato for the IT backbone supporting Flux data capture, MSL (Magnetic Shields Limited) for help with passive magnetic shielding, Jason Johnson for operations support, Husam Katnani for system design guidance, Dan Sobek for help with MEMS vapor cells, Mariano Troccoli for development of laser sources, John Kitching for early strategy discussions, Ryan Field for subsequent strategy discussions, and Bryan Johnson for helpful discussions and product vision.

\section{REFERENCES}

[1] Da Silva, F., Lopes, H., Hansen, P., Kringelbach, M., and Salmelin, R., [MEG: an introduction to methods], Oxford University Press, New York, 13-14, (2010).

[2] Okada, Y.C., Lahteenmäki, A. and Xu, C., "Experimental analysis of distortion of magnetoencephalography signals by the skull." Clinical neurophysiology 110 (2), 230-238 (1999). 
[3] Romani, G. L., Del Gratta, C. and Pizzella, V., "Neuromagnetism and its clinical applications." In [SQUID Sensors: Fundamentals, Fabrication and Applications], Springer, Dordrecht, 445-490 (1996).

[4] Robinson, J.T., Pohlmeyer, E., Gather, M.C., Kemere, C., Kitching, J.E., Malliaras, G.G., Marblestone, A., Shepard, K.L., Stieglitz, T. and Xie, C., "Developing Next-Generation Brain Sensing Technologies-A Review." IEEE sensors journal, 19(22), 10163-10175 (2019).

[5] Shah, V., Knappe, S., Schwindt, P.D. and Kitching, J., "Subpicotesla atomic magnetometry with a microfabricated vapour cell." Nature Photon 1, 649-652 (2007).

[6] Kitching, J., “Chip-scale atomic devices.” Applied Physics Reviews, 5(3), 031302 (2018).

[7] Hill, R.M., Boto, E., Rea, M., Holmes, N., Leggett, J., Coles, L.A., Papastavrou, M., Everton, S.K., Hunt, B.A.E., Sims, D. and Osborne J., "Multi-channel whole-head OPM-MEG: helmet design and a comparison with a conventional system." Neuroimage 219, 116995 (2020).

[8] Griffith, W.C., Knappe, S. and Kitching, J., "Femtotesla atomic magnetometry in a microfabricated vapor cell." Optics express 18, (26), 27167-27172 (2010).

[9] Boto, E., Holmes, N., Leggett, J., Roberts, G., Shah, V., Meyer, S.S., Muñoz, L.D., Mullinger, K.J., Tierney, T.M., Bestmann, S. and Barnes, G.R., "Moving magnetoencephalography towards real-world applications with a wearable system." Nature, 555(7698), 657-661 (2018).

[10] Sander, T.H., Preusser, J., Mhaskar, R., Kitching, J., Trahms, L., and Knappe, S., "Magnetoencephalography with a chip-scale atomic magnetometer." Biomedical Optics Express 3, (5), 981-990 (2012).

[11] Schneiderman J.F., Ruffieux S., Pfeiffer C., Riaz B. “On-Scalp MEG.” In: Supek S., Aine C. (eds) [Magnetoencephalography]. Springer, Cham. 1313-1335 (2019)

[12] Tierney, T.M., Holmes, N., Mellor, S., López, J.D., Roberts, G., Hill, R.M., Boto, E., Leggett, J., Shah, V., Brookes, M.J. and Bowtell, R., "Optically pumped magnetometers: From quantum origins to multi-channel magnetoencephalography." Neurolmage, 199, 598-608 (2019).

[13] livanainen, J., Zetter, R., Grön, M., Hakkarainen, K. and Parkkonen, L., "On-scalp MEG system utilizing an actively shielded array of optically-pumped magnetometers." Neuroimage 194, 244-258 (2019).

[14] livanainen, J., Stenroos, M. and Parkkonen, L., "Measuring MEG closer to the brain: Performance of on-scalp sensor arrays." Neurolmage 147, 542-553 (2017).

[15] Borna, A, Carter, T.R., Colombo, A.P., Jau, Y.Y., McKay, J., Weisend, M., Taulu, S., Stephen, J.M., and Schwindt, P.D., "Non-invasive functional-brain-imaging with an OPM-based magnetoencephalography system." Plos one 15 (1), (2020).

[16] Kitching, J., Knappe, S., Gerginov, V., Shah, V., Schwindt, P.D., Lindseth, B., Donley E.A., "Chip-scale atomic devices: precision atomic instruments based on MEMS." In Frequency Standards And Metrology, 445-453 (2009). 
[17] Kitching, J., Knappe, S. and Donley, E.A., "Atomic sensors-a review." IEEE Sensors Journal, 11(9), 1749-1758 (2011).

[18] Budker, D. and Romalis, M., “Optical magnetometry”. Nature physics, 3(4), 227-234 (2007).

[19] Dupont-Roc, J., Haroche, S. \& Cohen-Tannoudji, C., "Detection of very weak magnetic fields (10-9 gauss) by Rb zero-field level crossing resonances", Phys. Lett. A 28, 638-639 (1969).

[20] Happer, W., “Optical pumping”, Rev. Mod. Phys., 44 (2), 169-249 (1972).

[21] Purcell, E.M., Field, G.B., "Influence of collisions upon population of hyperfine states in hydrogen", Astrophys. J., 124, 542 (1956).

[22] Kominis, I.K., Kornack, T.W., Allred, J.C. and Romalis, M.V., "A subfemtotesla multichannel atomic magnetometer." Nature, 422(6932), 596-599 (2003).

[23] Ledbetter, M.P., Savukov, I.M., Acosta, V.M., Budker, D. and Romalis, M.V., "Spin-exchange-relaxation-free magnetometry with Cs vapor." Physical Review A, 77(3), 033408 (2008).

[24] Bloom, A. L., "Principles of operation of the rubidium vapor magnetometer." Applied Optics 1(1), 61-68 (1962).

[25] Bell, W.E., and Bloom, A.L., "Optically driven spin precession." Physical Review Letters 6, (6), 280 (1961).

[26] Roberts, G., Holmes, N., Alexander, N., Boto, E., Leggett, J., Hill, R.M., Shah, V., Rea, M., Vaughan, R., Maguire, E.A. and Kessler, K., "Towards OPM-MEG in a virtual reality environment." Neurolmage, 199, 408-417 (2019).

[27] Zhang, R., Xiao, W., Ding, Y., Feng, Y., Peng, X., Shen, L., Sun, C., Wu, T., Wu, Y., Yang, Y. and Zheng, Z., "Recording brain activities in unshielded Earth's field with optically pumped atomic magnetometers." Science Advances, 6(24) (2020).

[28] de Cheveigné, A., Wong, D.D., Di Liberto, G.M., Hjortkjaer, J., Slaney, M. and Lalor, E., “Decoding the auditory brain with canonical component analysis." Neurolmage, 172, 206-216 (2018).

[29] Mellinger, J., Schalk, G., Braun, C., Preissl, H., Rosenstiel, W., Birbaumer, N. and Kübler, A., "An MEG-based brain-computer interface (BCI)." Neuroimage, 36(3), 581-593 (2007).

[30] Wolpaw, J.R., McFarland, D.J., Neat, G.W. and Forneris, C.A., "An EEG-based brain-computer interface for cursor control." Electroencephalography and clinical neurophysiology, 78(3), 252-259 (1991).

[31] Lightfoot, G., "Summary of the N1-P2 cortical auditory evoked potential to estimate the auditory threshold in adults". Seminars in hearing, 37(1), 1 (2016).

[32] Virtanen, J., Ahveninen, J., Ilmoniemi, R. J., Näätänen, R., \& Pekkonen, E., "Replicability of MEG and EEG measures of the auditory N1/N1m-response." Electroencephalography and Clinical Neurophysiology/Evoked Potentials Section, 108(3), 291-298 (1998). 
[33] Gascoyne, L., Furlong, P. L., Hillebrand, A., Worthen, S. F., \& Witton, C., "Localising the auditory N1m with event-related beamformers: localisation accuracy following bilateral and unilateral stimulation." Scientific reports, 6(1), 1-9 (2016).

[34] Borna, A., Carter, T.R., Goldberg, J.D., Colombo, A.P., Jau, Y.Y., Berry, C., McKay, J., Stephen, J., Weisend, M. and Schwindt, P.D., "A 20-channel magnetoencephalography system based on optically pumped magnetometers." Physics in Medicine \& Biology, 62(23), 8909 (2017). 